\title{
On the Robustness of Global Optima and Stationary Solutions to Stochastic Mathematical Programs with Equilibrium Constraints, Part 1: Theory
}

\author{
C. Cromvik* M. Patriksson ${ }^{\dagger}$ \\ Communicated by F. Giannessi
}

${ }^{*}$ Mathematical Sciences, Chalmers University of Technology and Mathematical Sciences, University of Gothenburg, SE-412 96 Gothenburg, Sweden. E-mail: christoffer.cromvik@chalmers.se

${ }^{\dagger}$ Corresponding author. Mathematical Sciences, Chalmers University of Technology and Mathematical Sciences, University of Gothenburg, SE-412 96 Gothenburg, Sweden. E-mail: mipat@chalmers.se 
Abstract We consider a stochastic mathematical program with equilibrium constraints (SMPEC), and show that, under certain assumptions, global optima and stationary solutions are robust with respect to changes in the underlying probability distribution. In particular, the discretization scheme sample average approximation (SAA), which is convergent for both global optima and stationary solutions, can be combined with the robustness results to motivate the use of SMPECs in practice. We then study two new and natural extensions of the SMPEC model. First, we establish the robustness of global optima and stationary solutions to an SMPEC model where the upper-level objective is the risk measure known as conditional value-at-risk (CVaR). Second, we analyze a multiobjective SMPEC model, establishing the robustness of weakly Pareto optimal and weakly Pareto stationary solutions. In the accompanying paper (Cromvik and Patriksson, On the Robustness of Global Optima and Stationary Solutions to Stochastic Mathematical Programs with Equilibrium Constraints, part 2: Applications, Journal of Optimization Theory and Applications, 2010, to appear) we present applications of these results to robust traffic network design and to robust intensity modulated radiation therapy.

Keywords: Stochastic mathematical program with equilibrium constraints, Solution stability and robustness, Sample average approximation, Weak Pareto optimality 


\section{Introduction}

A physical system is often subjected to uncertainties, such as uncertain material parameters in a structural problem and stochastic market in an application to economics. Although it may be sufficient to consider a representative (e.g. mean) value of the uncertain parameters in order to produce a simulation of the system, the solution to an optimization problem that is based on its response can be very sensitive; using mean values of uncertain parameters can then give suboptimal solutions.

There are two main approaches to incorporating uncertainty into optimization models. In stochastic programming (see e.g. $[1,2]$ ), the objective function is an expected value, sometimes in combination with a risk measure. In such models, either all the decisions are made before any realization of the uncertain data, or the model contains a recourse opportunity so that some decisions can be made at a second stage. In robust optimization, an optimal solution is required to be feasible for all realizations of the uncertain data. The probability distributions utilized in stochastic programming are here replaced by the requirements that parameter values are confined to special bounded sets. Robust optimization provides a guarantee that an optimal solution is safe, but it is a pessimistic approach, since it considers the worst-case scenario. It is tractable for certain convex problems (see [3]); however, it has also been utilized for nonconvex problems through a linearization of the constraints ([4]).

We are interested in several applications which all can be modeled as a mathematical program with equilibrium constraints (MPEC). The stochastic extension of MPEC is a stochastic mathematical program with equilibrium constraints (SMPEC) ( [5]); the focus of this paper is to analyze the stability of optimal and stationary solutions to SMPEC when the underlying probability distribution is itself uncertain. This is motivated by practical applications such as the optimization of treatment plans in radiation therapy, where the biological response to radiation depends on parameters of which the probability distribution is uncertain; see our further discussions on this topic in [6].

We show that the SMPEC model is robust under certain conditions; we also show how to discretize a continuous distribution using sample average approximation (SAA) and that such an approximation will converge. Not surprisingly, similar conditions are required for robustness as for convergence of SAA.

The quantitative stability of solutions to stochastic programs due to changes in the probability distribution has been studied previously in [7] for general stochastic programs, for convex programs in [8], and for multistage programs in [9]; their focus is on the Lipschitz continuity of global optimal objective values. In contrast, our approach is qualitative in nature and provides stability results on optimal as well as stationary solutions. The subjects of optimality conditions and numerical methods for SMPECs have been studied previously, for example, in [10-12].

The remainder of this paper is organized as follows. In Section 2, we introduce the SMPEC model. In Section 3, we derive conditions under which global optima and stationary solutions are stable with respect to perturbations in the probability distribution. In Section 4, we present an extension of the SMPEC to include the risk measure CVaR, and establish the robustness of its global optima and stationary solutions. In Section 5, we present a discretization scheme, and show that it converges when the discretization 
is refined. Combining stability with a convergent discretization scheme provides a motivation for the use of SMPEC in practice. In Section 6, we extend the stability result to SMPECs with multiple objectives. Finally, in Section 7, we provide a summary and future research opportunities.

\section{Stochastic Mathematical Programs with Equilib- rium Constraints}

\subsection{Mathematical Programs with Equilibrium Constraints}

Consider a mathematical program with an equilibrium constraint in the form of a variational inequality,

$$
\begin{array}{lll}
(\mathrm{MPEC}) & \min _{(x, y)} & f(x, y), \\
\text { s.t. } & x \in X, \\
& & -F(x, y) \in N_{C}(y),
\end{array}
$$

where $f: \mathbb{R}^{n} \times \mathbb{R}^{m} \rightarrow \mathbb{R}, y \in \mathbb{R}^{m}, C \subseteq \mathbb{R}^{m}$ is a polyhedron, $F(x, \cdot): C \rightarrow \mathbb{R}^{m}$ is smooth, and $N_{C}: \mathbb{R}^{m} \rightrightarrows \mathbb{R}^{m}$ is the standard normal cone mapping

$$
N_{C}(y):= \begin{cases}\left\{z \in \mathbb{R}^{m} \mid z^{\mathrm{T}}(w-y) \leq 0, w \in C\right\}, & \text { if } y \in C, \\ \emptyset, & \text { otherwise. }\end{cases}
$$

The vector $x \in \mathbb{R}^{n}$ represents the design (or primary) variables and $y \in \mathbb{R}^{m}$ is the response (or secondary) variables. The nonempty, closed and convex set $X \subseteq \mathbb{R}^{n}$ specifies the set of feasible designs. Note that there are no joint upper-level constraints in this setting, which is natural when considering the stability of optimal solutions (cf. [13-15]).

The variational inequality, $-F(x, y) \in N_{C}(y)$, can represent an equilibrium in a general form. For example, with $C=\mathbb{R}^{m}$, the variational inequality is equivalent to the system of equations

$$
F(x, y)=0^{m},
$$

and with $C=\mathbb{R}_{+}^{m}$, the variational inequality is equivalent to the complementarity constraint

$$
0^{m} \leq y \perp F(x, y) \geq 0^{m},
$$

where $a \perp b$ means that $a^{\mathrm{T}} b=0$. Since complementarity constraints are examples of equilibrium constraints, it indicates that MPECs are usually very nonlinear and irregular. In fact, MPECs lack standard constraint qualifications ( [16]), which can highly influence the performance of nonlinear optimization algorithms for solving MPECs. For recent work on the numerical solution of MPECs, we refer to $[17,18]$.

If $F(x, y)=\nabla_{y} \phi(x, y)$ for a $C^{1}$ function $\phi(x, \cdot)$, then the variational inequality

$$
\nabla \phi(x, y)^{\mathrm{T}}(\bar{y}-y) \geq 0, \quad \forall \bar{y} \in C,
$$

represents the optimality conditions for the parametric optimization problem

$$
\min _{y \in C} \phi(x, y),
$$


and the MPEC becomes what is traditionally known as a bilevel optimization problem ( [19]).

The generality of the variational inequality suggests that a number of optimization problems can be put into the form of (MPEC) (see [20-22]). For example, the Stackelberg game [23], which is a leader-follower game and an extension of the Nash game [24], can be formulated as an MPEC. The accompanying paper [6] numerically analyzes applications to the design of traffic networks and optimal treatment plans in intensity-modulated radiation therapy (IMRT).

\subsection{Stochastic Mathematical Program with Equilibrium Con- straints}

Next, we consider the stochastic extension of (MPEC). Let $(\Omega, \Theta, P)$ be a complete probability space and consider the problem

$$
\begin{aligned}
\left(\mathrm{SMPEC}_{\Omega}\right) \min _{(x, y(\cdot))} & \mathrm{E}_{\omega}[f(x, y(\omega), \omega)]:=\int_{\Omega} f(x, y(\omega), \omega) P(d \omega), \\
\text { s.t. } \quad & x \in X, \\
& -F(x, y, \omega) \in N_{C}(y), \quad P \text {-a.s., }
\end{aligned}
$$

where $y: \Omega \rightarrow \mathbb{R}^{m}$ is a random element of the probability space $(\Omega, \Theta, P)$. We also introduce $S: \mathbb{R}^{n} \times \Omega \rightrightarrows \mathbb{R}^{m}$, which defines the set of solutions to the lower-level parametric variational inequality problem,

$$
S(x, \omega):=\left\{y \in \mathbb{R}^{m} \mid-F(x, y, \omega) \in N_{C}(y)\right\} .
$$

In view of stochastic programming with recourse, SMPEC is considered as a here-and-now type of problem, where decision $x$ should be taken before any realizations of uncertain data.

When the solution to the lower-level problem is nonunique for a fixed $x$ and $\omega$, the model should be interpreted as $y$ being chosen such that the objective function is minimized given $x$ and $\omega$.

This formulation of SMPEC follows the original one of Patriksson and Wynter [5] and of Evgrafov and Patriksson [25]. Alternative formulations are found in [10,11,26].

\subsection{Existence of Optimal Solutions}

The following assumption will be in force throughout this paper:

\section{Assumption A}

(A1) The mapping $S(x, \cdot)$ is measurable for every $x$.

(A2) The set $X$ is closed and the mapping $x \mapsto S(x, \omega)$ is closed for almost every $\omega \in \Omega$.

(A3) The function $f$ is continuous in $(x, y)$, measurable in $\omega$, uniformly weakly coercive with respect to $x$ over the set $X$, and bounded from below by a $(\Theta, \mathrm{P})$-integrable function. 
(A4) The set $S\left(x_{0}, \omega\right)$ is nonempty for some $x_{0} \in X$ and almost every $\omega \in \Omega$.

The following result on the existence of optimal solutions is due to Evgrafov and Patriksson [25].

Theorem 2.1 (Existence of Optimal Solutions) Let Assumption (A) hold. Then, problem $\left(\mathrm{SMPEC}_{\Omega}\right)$ has at least one optimal solution.

\section{Solution Stability}

We are interested in the stability of optimal solutions to $\left(\mathrm{SMPEC}_{\Omega}\right)$ with respect to changes in the probability distribution. Such results have value both from a computational and a theoretical viewpoint. If the problem is stable, then it can be approximated by using discrete probability measures, resulting in a finite-dimensional problem. From a theoretical point of view, we deduct that the problem is robust. We analyze the stability of globally optimal solutions (Theorem 3.1) as well as of stationary solutions (Theorem $3.2)$.

\subsection{Stability of Globally Optimal Solutions}

We first analyze the stability of globally optimal solutions. This is foremost motivated by simplicity. For global optima, we can relate the perturbations of the probability distribution to changes in the objective value. For stationary solutions, this becomes more problematic.

The result of stability of globally optimal solutions is particularly interesting for convex problems, where we can find global optima in practice.

Let $\left\{P_{k}\right\}$ be a sequence of probability measures defined on $\mathcal{B}(\Omega)$. Consider the associated sequence of optimization problems,

$$
\begin{aligned}
\left(\mathrm{SMPEC}_{\Omega}\right)^{k} \quad \min _{(x, y(\cdot))} & \mathrm{E}_{\omega}[f(x, y(\omega), \omega)]:=\int_{\Omega} f(x, y(\omega), \omega) P_{k}(d \omega), \\
\text { s.t. } \quad & x \in X, \\
& -F(x, y, \omega) \in N_{C}(y), \quad P_{k} \text {-a.s. }
\end{aligned}
$$

The problem differs from $\left(\mathrm{SMPEC}_{\Omega}\right)$ only in the choice of probability distribution. Let $\operatorname{val}(P)$ denote the optimal value of problem $P$. The following result shows the stability of globally optimal solutions. The corresponding result in the context of topology optimization in structural mechanics can be found in [15] and for network design under traffic equilibrium in $[27,28]$. The proof presented here is similar.

Theorem 3.1 (Global Stability of Optimal Solutions to $\left(\mathrm{SMPEC}_{\Omega}\right)$ ) Let Assumption (A) hold, suppose that the mapping $F(x, \cdot, \omega)$ is strictly monotone in $y$ for each $x \in X$ and $\omega \in \Omega$, and that the sequence $\left\{P_{k}\right\}$ of probability measures weakly converges to $P$. Also, suppose that, for each $k,\left(x^{k}, y^{k}(\cdot)\right)$ solves $\left(\mathrm{SMPEC}_{\Omega}\right)^{k}$. Then, each limit point (there is at least one) of the sequence $\left\{\left(x^{k}, y^{k}(\cdot)\right)\right\}$ is an optimal solution to $\left(\mathrm{SMPEC}_{\Omega}\right)$. 
Proof. Consider an optimal solution $\left(x^{*}, y^{*}(\cdot)\right)$ to $\left(\mathrm{SMPEC}_{\Omega}\right)$. Since $F$ is strictly monotone in $y, y$ is continuous in $x$ and $\omega$. By (A3), any sequence of feasible designs and responses is bounded and hence has a limit point. The optimal solution to $\left(\operatorname{SMPEC}_{\Omega}\right)$ is moreover feasible in $\left(\operatorname{SMPEC}_{\Omega}\right)^{k}$ for all $k$. It follows that val $\left(\operatorname{SMPEC}_{\Omega}\right) \geq$ $\lim \sup _{k \rightarrow \infty} \operatorname{val}\left(\mathrm{SMPEC}_{\Omega}\right)^{k}$.

Next, let $\left\{\left(x^{k}, y^{k}(\cdot)\right)\right\}$ be a sequence of optimal solutions to $\left(\mathrm{SMPEC}_{\Omega}\right)^{k}$. By (A3), this sequence is bounded. Denote any limit point $(\bar{x}, \bar{y}(\cdot))$. It is feasible for almost every $\omega$ in $\left(\mathrm{SMPEC}_{\Omega}\right)$. Using the lower semicontinuity of $f$ and Fatou's lemma, we get

$$
\begin{aligned}
\operatorname{val}\left(\operatorname{SMPEC}_{\Omega}\right) & \leq \int_{\Omega} f(\bar{x}, \bar{y}(\omega), \omega) p(\omega) d \omega \\
& \leq \int_{\Omega} \liminf _{k \rightarrow \infty} f\left(x^{k}, y^{k}(\omega), \omega\right) p(\omega) d \omega \\
& \leq \liminf _{k \rightarrow \infty} \int_{\Omega} f\left(x^{k}, y^{k}(\omega), \omega\right) p_{k}(\omega) d \omega \\
& =\liminf _{k \rightarrow \infty} \operatorname{val}\left(\operatorname{SMPEC}_{\Omega}\right)^{k} .
\end{aligned}
$$

By combining the two inequalities, we get the result.

\subsection{Stability of Stationary Solutions}

Due to the nonconvex nature of MPECs, it is not reasonable in general to expect algorithms to find globally optimal solutions. This fact limits the practical use of Theorem 3.1 , and raises the question of stability of stationary solutions. The proof of stability for globally optimal solutions was based on analyzing the convergence of the optimal value. For stationary solutions, we need to analyze the conditions of (local) optimality which relates to stationarity.

Optimality conditions for $\left(\mathrm{SMPEC}_{\Omega}\right)$ are nontrivial to formulate due to the presence of the variational inequality. Under certain conditions, the response variable $y$ can be treated as an implicit variable; this reduces the complexity of formulating optimality conditions for the SMPEC. This technique is used by Outrata [21] for the MPEC, which has inspired the proof approach below.

The following assumption will be utilized in addition to Assumption A.

\section{Assumption B}

(B1) The function $f$ is Lipschitz continuous in $(x, y)$.

(B2) The mapping $F(\cdot, \cdot, \omega)$ is continuously differentiable and $F(x, \cdot, \omega)$ is uniformly strongly monotone on $C$ for each $x \in X$ and $\omega \in \Omega$, i.e.,

$$
\left(F\left(x, y_{1}, \omega\right)-F\left(x, y_{2}, \omega\right)\right)^{\mathrm{T}}\left(y_{1}-y_{2}\right) \geq c\left\|y_{1}-y_{2}\right\|^{2}, \quad \forall y_{1}, y_{2} \in C,
$$

where $c>0$ is independent of $x$ and $\omega$.

(B3) $X=\left\{x \in \mathbb{R}^{n} \mid g_{i}(x) \leq 0, i=1, \ldots, p\right\}$ and each function $g_{i}$ is continuously differentiable; 
(B4) The Mangasarian-Fromovitz constraint qualification (MFCQ) holds for all $x \in X$.

If Assumptions (B1) and (B2) hold, then (see [29]) there exists a locally Lipschitz continuous, single-valued solution map $(x, \omega) \mapsto \sigma(x, \omega)$ with

$$
y=\sigma(x, \omega), \quad \sigma(x, \omega) \in S(x, \omega) .
$$

With this property, we can rewrite $\left(\mathrm{SMPEC}_{\Omega}\right)$ as the one-level problem,

$$
\begin{aligned}
\left(\operatorname{SNLP}_{\Omega}\right) \quad \min _{x} \quad \mathrm{E}_{\omega}[f(x, \sigma(x, \omega), \omega)]:=\int_{\Omega} f(x, \sigma(x, \omega), \omega) P(d \omega), \\
\text { s.t. } \quad x \in X
\end{aligned}
$$

and correspondingly $\left(\mathrm{SNLP}_{\Omega}\right)^{k}$ is obtained from $\left(\mathrm{SMPEC}_{\Omega}\right)$ by replacing $P$ with $P_{k}$.

Before stating the optimality conditions, we introduce two definitions from nonsmooth analysis.

Definition 3.1 The Clarke directional derivative of a function $f: \mathbb{R}^{n} \rightarrow \mathbb{R}$ at $x$ in the direction $h$ is defined by

$$
f^{0}(x ; h):=\limsup _{\substack{t \downarrow 0 \\ z \rightarrow x}} \frac{f(z+t h)-f(z)}{t} .
$$

Since $f$ is Lipschitz continuous [Assumption $(\mathrm{B} 1)], f^{0}(\cdot, h)$ is upper semicontinuous ( [30, Proposition 2.1.1]).

Definition 3.2 The generalized gradient of $f$ at $x$ is defined as the set

$$
\partial f(x):=\left\{\xi \in \mathbb{R}^{n} \mid(\xi, h) \leq f^{0}(x ; h)\right\} .
$$

If $f$ is continuously differentiable at $x$ then $\partial f(x)=\{\nabla f(x)\}$. If Assumptions (B3) and (B4) hold, a vector $x^{*} \in X$ is a Clarke stationary solution (see [30, Theorem 6.1.1], see $\left[31\right.$, Theorem 6.1.8]) to $\left(\operatorname{SNLP}_{\Omega}\right)$ if, for some vector $\mu \in \mathbb{R}_{+}^{p}$ with $\mu_{i} g_{i}\left(x^{*}\right)=0$ for all $i$, we have

$$
\left.0^{n} \in \partial \mathrm{E}_{\omega}\left[f\left(x^{*}, \sigma\left(x^{*}, \omega\right), \omega\right)\right]\right)+\nabla g\left(x^{*}\right) \mu,
$$

where $\mu$ is the vector of Lagrange multipliers. Analogously, a vector $x^{*} \in X$ is a weakly Clarke stationary solution if,

$$
\left.0^{n} \in \mathrm{E}_{\omega}\left[\partial f\left(x^{*}, \sigma\left(x^{*}, \omega\right), \omega\right)\right]\right)+\nabla g\left(x^{*}\right) \mu .
$$

Theorem 3.2 (Stability of Stationary Solutions to $\left(\mathrm{SNLP}_{\Omega}\right)$ ) Let Assumption (A) and (B) hold, suppose that the sequence $\left\{P_{k}\right\}$ of probability measures weakly converges to $P$ and is upper bounded by a measurable function, and that, for each $k,\left(x^{k}, y^{k}(\cdot)\right)$ is a Clarke stationary solution to $\left(\mathrm{SNLP}_{\Omega}\right)^{k}$. Then, each limit point (there is at least one) of the sequence $\left\{\left(x^{k}, y^{k}(\cdot)\right)\right\}$ is a weakly Clarke stationary solution to $\left(\operatorname{SNLP}_{\Omega}\right)$. 
Proof. By the assumptions, $F(x, \cdot, \omega)$ is uniformly strongly monotone, and therefore the solution map $S(x, \omega)$ is single-valued and Lipschitz continuous (cf. [29]). This enables us to use the one-level problems $\left(\operatorname{SNLP}_{\Omega}\right)$ and $\left(\operatorname{SNLP}_{\Omega}\right)^{k}$. Since $\sigma$ and $f$ are Lipschitz continuous, there exists a random variable $k(\omega) \geq 0$ such that $\mathrm{E}[k(\omega)]<\infty$ and such that, for all $x_{1}, x_{2} \in X$,

$$
\left|f\left(x_{1}, \sigma\left(x_{1}, \omega\right), \omega\right)-f\left(x_{2}, \sigma\left(x_{2}, \omega\right), \omega\right)\right| \leq k(\omega)\left\|x_{1}-x_{2}\right\| .
$$

Let $x^{k}$ be a Clarke stationary solution to $\left(\operatorname{SNLP}_{\Omega}\right)^{k}$ and consider a sequence $\left\{x^{k}\right\}$ of such stationary solutions. Since $f$ is inf-compact [see Assumption (A3)], this sequence is bounded. Denote a limit point by $x^{*}$. Define

$$
\mathrm{E}_{\omega}^{k}[f(x)]:=\int_{\Omega} f(x, \sigma(x, \omega), \omega) P_{k}(d \omega) .
$$

The point $x^{k}$ is stationary if

$$
0^{n} \in \partial \mathrm{E}_{\omega}^{k}\left[f\left(x^{k}\right)\right]+\nabla g\left(x^{k}\right) \mu^{k}
$$

and

$$
0^{p} \leq \mu^{k} \perp g\left(x^{k}\right) \leq 0^{p} .
$$

Fix a direction $h \in \mathbb{R}^{n}$. Then, we have

$$
\begin{aligned}
\left(\mathrm{E}_{\omega}^{k}[f]\right)^{0}(x ; h) & =\limsup _{\substack{t \downarrow 0 \\
z \rightarrow x}} \frac{\mathrm{E}_{\omega}^{k}[f(z+t h)]-\mathrm{E}_{\omega}^{k}[f(z)]}{t} \\
& =\limsup _{\substack{t \downarrow 0 \\
z \rightarrow x}} \frac{\int_{\Omega} f(z+t h, \sigma(z+t h, \omega), \omega) P_{k}(d \omega)-\int_{\Omega} f(z, \sigma(z, \omega), \omega) P_{k}(d \omega)}{t} \\
& =\limsup _{\substack{t \downarrow 0 \\
z \rightarrow x}} \int_{\Omega} \frac{(f(z+t h, \sigma(z+t h, \omega), \omega)-f(z, \sigma(z, \omega), \omega))}{t} P_{k}(d \omega) \\
& \leq \int_{\Omega} f^{0}(x, \sigma(x, \omega), \omega ; h) P_{k}(d \omega)=E_{\omega}^{k}\left[f^{0}(x, \sigma(x, \omega), \omega ; h)\right],
\end{aligned}
$$

where the last inequality follows by Equation (1), with $x_{1}=z$ and $x_{2}=z+t h$, and the Lebesgue dominated convergence theorem. Furthermore, we have that

$$
\begin{aligned}
\limsup _{k \rightarrow \infty} E_{\omega}^{k}\left[f^{0}\left(x^{k} ; h\right)\right] & =\limsup _{k \rightarrow \infty} \int_{\Omega} f^{0}\left(x^{k}, \sigma\left(x^{k}, \omega\right), \omega ; h\right) P_{k}(d \omega) \\
& \leq \int_{\Omega} \limsup _{k \rightarrow \infty} f^{0}\left(x^{k}, \sigma\left(x^{k}, \omega\right), \omega ; h\right) P_{k}(d \omega) \\
& \leq \int_{\Omega} f^{0}\left(x^{*}, \sigma\left(x^{*}, \omega\right), \omega ; h\right) P(d \omega) \\
& =E_{\omega}\left[f^{0}\left(x^{*} ; h\right)\right],
\end{aligned}
$$

where we use the Lebesgue dominated convergence theorem in the second equality and the upper semicontinuity of $f^{0}$ in the second inequality. Hence,

$$
\limsup _{k \rightarrow \infty} \partial \mathrm{E}_{\omega}^{k}\left[f\left(x^{k}\right)\right] \subset \mathrm{E}_{\omega}\left[\partial f\left(x^{*}\right)\right] .
$$


Next, we argue that

$$
\limsup _{k \rightarrow \infty} \nabla g\left(x^{k}\right) \mu^{k}=\nabla g\left(x^{*}\right) \mu^{*} .
$$

Suppose that this is not true, but $\left\|\mu^{k}\right\| \rightarrow \infty$. We can then define $\lambda^{k}:=\mu^{k} /\left\|\mu^{k}\right\|$, and assume that $\lambda^{k} \rightarrow \lambda^{*}$ for some $\lambda^{*}$ such that

$$
\lambda^{*} \geq 0^{p},\left\|\lambda^{*}\right\|=1
$$

Let $I(x):=\left\{i \mid g_{i}(x)=0\right\}$ be the set of active constraint indices at $x$. By the definition of MFCQ, to each $x^{k}$, there exists a vector $d \in \mathbb{R}^{n}$ such that

$$
\begin{array}{ll}
\nabla g_{i}\left(x^{k}\right)^{\mathrm{T}} d<0, & i \in I\left(x^{k}\right), \\
g_{i}\left(x^{k}\right)<0, & i \notin I\left(x^{k}\right),
\end{array}
$$

For $i \in I\left(x^{*}\right)$ we must have $\lambda_{i}^{k} \rightarrow 0$. Fix a $d \in \mathbb{R}^{n}$ such that a condition like (4) holds at $x^{*}$. Then, by (2), we have

$$
\begin{aligned}
0 & \leq \limsup _{k \rightarrow \infty}\left(\frac{d^{\mathrm{T}} \nabla g\left(x^{k}\right) \mu_{k}}{\left\|\mu_{k}\right\|}+\frac{\left(E_{\omega}^{k}[f]\right)^{0}\left(x^{k} ; d\right)}{\left\|\mu_{k}\right\|}\right)=\limsup _{k \rightarrow \infty} d^{\mathrm{T}} \nabla g\left(x^{k}\right) \lambda^{k} \\
& =\sum_{i \in I\left(x^{*}\right)} \lambda_{i}^{*} \nabla g_{i}\left(x^{*}\right)^{\mathrm{T}} d .
\end{aligned}
$$

From the last expression and (4), we get

$$
0 \leq \sum_{i \in I\left(x^{*}\right)} \lambda_{i}^{*} \nabla g_{i}\left(x^{*}\right)^{\mathrm{T}} d \leq-\sum_{i \in I\left(x^{*}\right)} \lambda_{i}^{*},
$$

and since $\lambda^{*} \geq 0^{p}$, this implies that $\lambda^{*}=0$. This contradicts the assumption $\left\|\lambda^{*}\right\|=1$, and so $\mu^{*}$ must be bounded.

To sum up, we have

$$
0 \in \limsup _{k \rightarrow \infty}\left(\partial \mathrm{E}_{\omega}^{k}\left[f\left(x^{k}\right)\right]+\nabla g\left(x^{k}\right) \mu^{k}\right) \subset \mathrm{E}_{\omega}\left[\partial f\left(x^{*}\right)\right]+\nabla g\left(x^{*}\right) \mu^{*}
$$

and we can therefore conclude that $x^{*}$ is a weakly Clarke stationary solution to $\left(\mathrm{SNLP}_{\Omega}\right)$. $\square$

If $f$ is regular (see Assumption (C2)), then (see [30, Theorem 2.7.2]) we have

$$
\mathrm{E}[\partial f(x)]=\partial \mathrm{E}[f(x)]
$$

in the above theorem, and we may then make the stronger conclusion that the sequence $\left\{x^{k}\right\}$ of Clarke stationary solutions to $\left(\mathrm{SNLP}_{\Omega}\right)^{k}$ converges to a Clarke stationary solution to $\left(\mathrm{SNLP}_{\Omega}\right)$.

\section{Risk Objective Function}

In this section, we assume that the objective function $f(x, y(\omega), \omega)$ measures a loss. The value-at-risk ( $\mathrm{VaR})$ at probability level $\beta$ is denoted by $\beta$ - $\operatorname{VaR}(x)$; it is the value for which the probability that $f$ do not exceed this value is $\beta$, i.e.,

$$
\beta-\operatorname{VaR}(x)=\min \{\gamma \mid P(f(x, y(\omega), \omega) \leq \gamma) \geq \beta\} .
$$


Furthermore, the conditional value-at-risk, denoted $\beta$-CVaR $(x)$, is the conditional expectation of loss, given that the loss is greater than $\beta$-VaR, i.e.,

$$
\beta-\operatorname{CVaR}(x)=\frac{1}{1-\beta} \int_{f(x, y(\omega), \omega) \geq \beta-\operatorname{Var}} f(x, y(\omega), \omega) P(d \omega) .
$$

The parameter $\beta$ determines the level of risk. If $\beta=1, \mathrm{CVaR}$ equals the expected value; if $\beta=0, \mathrm{CVaR}$ equals the maximal value of $f$; the $\mathrm{CVaR}$ formalism therefore introduces the possibility to include interesting compromises between these two extremes.

Rockafellar and Uryasev [32] provide an alternative expression for $\beta$-CVaR which utilizes the following function:

$$
G_{\beta}(x, y, \gamma)=\gamma+\frac{1}{1-\beta} \int_{\Omega}[f(x, y(\omega), \omega)-\gamma]_{+} P(d \omega),
$$

where, for $s \in \mathbb{R},[s]_{+}:=\max \{0, s\}$. The conditional value-at-risk is equal to the minimum value of $G_{\beta}$ over $\gamma \in \mathbb{R}$, i.e.,

$$
\beta-\operatorname{CVaR}(x)=\min _{\gamma \in \mathbb{R}} G_{\beta}(x, y, \gamma) .
$$

The value-at-risk is a minimizer of $G_{\beta}$, and the problem of minimizing $\beta$-CVaR over $(x, y)$ is equivalent to minimizing $G_{\beta}$ over $(x, y, \gamma)([32])$. Note also that $G_{\beta}$ is convex in $\gamma$, so CVaR preserves convexity.

Consider now the SMPEC where the expected value in the objective function is replaced by the alternative expression for $\beta$-CVaR:

$$
\begin{aligned}
\left(\mathrm{SRPEC}_{\Omega}\right) \min _{(x, y(\cdot), \gamma)} & \gamma+\frac{1}{1-\beta} \int_{\Omega}[f(x, y(\omega), \omega)-\gamma]_{+} P(d \omega), \\
\text { s.t. } \quad & x \in X, \\
& -F(x, y, \omega) \in N_{C}(y), \quad P \text {-a.s. }
\end{aligned}
$$

We will next show that the robustness results for global optima (Theorem 3.1) and for stationary solutions (Theorem 3.2) can be extended to the SRPEC. Following the procedure in Section 3, let $\left\{P_{k}\right\}$ be a sequence of probability measures defined on $\mathcal{B}(\Omega)$ and consider the associated sequence of optimization problems $\left(\mathrm{SRPEC}_{\Omega}\right)^{k}$ which are obtained from $\left(\mathrm{SRPEC}_{\Omega}\right)$ by replacing $P$ with $P_{k}$.

Theorem 4.1 (Global Stability of Optimal Solutions to $\left(\mathrm{SRPEC}_{\Omega}\right)$ ) Let Assumption (A) hold, suppose that the mapping $F(x, \cdot, \omega)$ is strictly monotone in $y$ for each $x \in X$ and $\omega \in \Omega$, and that the sequence $\left\{P_{k}\right\}$ of probability measures weakly converges to $P$. Also suppose that, for each $k,\left(x^{k}, y^{k}(\cdot), \gamma^{k}\right)$ solves $\left(\mathrm{SRPEC}_{\Omega}\right)^{k}$. Then, each limit point (there is at least one) of the sequence $\left\{\left(x^{k}, y^{k}(\cdot), \gamma\right)\right\}$ is an optimal solution to $\left(\mathrm{SRPEC}_{\Omega}\right)$.

Proof. The proof follows essentially from that of Theorem 3.1. Two critical steps need to be motivated. The first is the continuity of the objective function. The function in the integral is continuous with respect to $x$ and $y$, since it is a decomposition of $f$ and $[\cdot]_{+}$, and since $F$ is strictly monotone. Also, the objective function $G_{\beta}$ is continuous with respect to $\gamma$. 
The second step is that the objective function is weakly coercive with respect to $(x, y, \gamma)$. This holds by Assumption (A3) and the fact that, if $|\gamma| \rightarrow \infty, G_{\beta} \rightarrow \infty$.

To establish robustness for stationary solutions, we again utilize the reformulation of SMPEC into one-level problems. We consider the following problem:

$$
\begin{aligned}
& \left(\operatorname{SRNLP}_{\Omega}\right) \quad \min _{(x, \gamma)} \gamma+\frac{1}{1-\beta} \int_{\Omega}[f(x, \sigma(x, \omega), \omega)-\gamma]_{+} P(d \omega) \\
& \text { s.t. } x \in X \text {. }
\end{aligned}
$$

Correspondingly, $\left(\operatorname{SRNLP}_{\Omega}\right)^{k}$ is obtained from $\left(\operatorname{SRNLP}_{\Omega}\right)$ by replacing $P$ with $P_{k}$.

Theorem 4.2 (Stability of Stationary Solutions to $\left(\operatorname{SRNLP}_{\Omega}\right)$ ) Let Assumption $(\mathrm{A})$ and (B) hold, suppose that the sequence $\left\{P_{k}\right\}$ of probability measures weakly converges to $P$ and is upper bounded by a measurable function and that, for each $k,\left(x^{k}, y^{k}(\cdot), \gamma^{k}\right)$ is a Clarke stationary solution to $\left(\mathrm{SRNLP}_{\Omega}\right)^{k}$. Then, each limit point (there is at least one) of the sequence $\left\{\left(x^{k}, y^{k}(\cdot), \gamma\right)\right\}$ is a weakly Clarke stationary solution to $\left(\mathrm{SRNLP}_{\Omega}\right)$.

Proof. The proof follows from that of Theorem 3.2, the proof of Theorem 4.1, and the fact that $G_{\beta}$ is Lipschitz continuous.

\section{Convergence of a Discretization Scheme}

In this section, we discuss the numerical solution of $\left(\mathrm{SMPEC}_{\Omega}\right)$. The objective function is a multidimensional integral which must be approximately computed in the general case. If it is discretized, it is natural to analyze the convergence of a discretization scheme. Having established both the stability of optimal solutions and the convergence of a numerical scheme puts us closer to the practical use of an SMPEC model.

One approach used extensively to numerically solve stochastic programs is a Monte Carlo technique known as sample average approximation (SAA) (see e.g. [33]). The idea is to draw $N$ iid samples $\omega^{1}, \ldots, \omega^{N}$ and solve a deterministic problem for increasing values of $N$. We use the reformulation of $(\mathrm{SMPEC})_{\Omega}$ into $\left(\mathrm{SNLP}_{\Omega}\right)$ and consider the problem

$$
\begin{aligned}
(\mathrm{SNLP})^{N} & \min _{x} \quad \hat{f}_{N}:=\frac{1}{N} \sum_{k=1}^{N} f\left(x, \sigma\left(x, \omega^{k}\right), \omega^{k}\right), \\
\text { s.t. } \quad & x \in X .
\end{aligned}
$$

To establish convergence as $N \rightarrow \infty$, the following additional conditions are required.

\section{Assumption C}

(C1) The set $X$ is bounded and convex.

(C2) The function $f(\cdot, \sigma(\cdot, \omega), \omega)$ is regular (i.e., $f$ is directionally differentiable and the directional derivative coincides with the Clarke directional derivative) at $x$ for almost every $\omega \in \Omega$. 
Condition (C2) is fulfilled if e.g. $f(\cdot, \sigma(\cdot, \omega), \omega)$ is convex or continuously differentiable ( [30]).

The convergence proofs for both globally optimal solutions and stationary solutions are based on the law of large numbers. The main difference in the assumptions needed is the requirement of a regular function for the convergence of stationary solutions.

Theorem 5.1 (Convergence of Optimal Solutions to $\left.\left(\operatorname{SNLP}_{\Omega}\right)\right)$ Let Assumptions (A), (B1)(B2), (C1) hold. For each $N$, let $\left(x_{N}, y_{N}(\cdot)\right)$ be an optimal solution to $(\mathrm{SNLP})^{N}$. Then, each limit point (there is at least one) of the sequence $\left\{x_{N}\right\}$ is an optimal solution to $\left(\mathrm{SNLP}_{\Omega}\right)$.

Proof. The feasible set is compact by Assumptions (A2) and (C1); by Assumptions (B1)-(B2), for almost every $\omega \in \Omega$ the objective function $f(\cdot, \cdot, \omega)$ is continuous. By Assumption (B2), it is also bounded from above by a $(\Theta, P)$-integrable function and the sample is iid. Then, by [33, Proposition 7], $\hat{f}_{N}$ converges to $f$ w.p. 1 uniformly on $X$. In turn, by [33, Proposition 5], this implies that $\operatorname{val}\left((\mathrm{SNLP})^{N}\right) \rightarrow \operatorname{val}\left((\mathrm{SNLP})_{\Omega}\right)$ as $N \rightarrow \infty$.

Theorem 5.2 (Convergence of Stationary Solutions to $\left(\operatorname{SNLP}_{\Omega}\right)$ ) Let Assumptions (A), (B), (C) hold. For each $N$, let $\left(x_{N}, y_{N}(\cdot)\right)$ be a stationary solution to $(\mathrm{SNLP})^{N}$. Then, each limit point (there is at least one) of the sequence $\left\{x_{N}\right\}$ is a stationary solution to $\left(\operatorname{SNLP}_{\Omega}\right)$.

Proof. By Assumptions (A3) and (B2), the objective function is of Carathéodory type; by Assumption ( $\mathrm{C} 1)$, the set is compact and convex. By $(\mathrm{C} 2)$, the function is also regular. Then, by [34, Theorem 7], the sequence of stationary solutions $\left\{x^{N}\right\}$ converges w.p.1 to a stationary solution of $\left(\mathrm{SNLP}_{\Omega}\right)$.

Since $\left(\mathrm{SNLP}_{\Omega}\right)$ is a reformulation of $\left(\mathrm{SMPEC}_{\Omega}\right)$, the above theorems state that we also have convergence to global optima and stationary solutions for the corresponding discretized problem

$$
\begin{aligned}
(\mathrm{SMPEC})^{N} \quad \min _{x} \quad \hat{f}_{N}:=\frac{1}{N} \sum_{k=1}^{N} f\left(x, y^{k}, \omega^{k}\right), \\
\text { s.t. } \quad x \in X, \\
\quad-F\left(x, y^{k}, \omega^{k}\right) \in N_{C}\left(y^{k}\right), \quad k=1, \ldots, N .
\end{aligned}
$$

To summarize, the results from Theorems 5.1 and 5.2 show that it is a valid approach to compute a solution to the SMPEC model through a sequence of deterministic problems. Theorems 5.1 and 5.2 are also immediate to extend to the CVaR model of Section 4.

Analogous discretization schemes for engineering applications are studied in Evgrafov and Patriksson $[14,15]$. 


\section{Stochastic Multiple Objective Mathematical Pro- grams with Equilibrium Constraints}

With $q$ objectives $f_{i}: X \rightarrow \mathbb{R}, i=1, \ldots, q$, the standard multiobjective optimization problem is

$$
\min _{x \in X}\left(f_{1}(x), \ldots, f_{q}(x)\right) .
$$

Let $f$ denote the $q$-vector of functions $f_{i}, i=1, \ldots, q$. We recall the definitions of Pareto and weakly Pareto optimal solutions.

Definition 6.1 A vector $\bar{x} \in X$ is called Pareto optimal if there is no $x \in X$ such that $f(x) \leq f(\bar{x})$ and $f_{i}(x)<f_{i}(\bar{x})$ for at least one $i=1, \ldots, q$. A feasible solution $\hat{x}$ is called weakly Pareto optimal if there is no $x \in X$ such that $f(x)<f(\hat{x})$.

The study of a multiple objective SMPEC problem appears to be new; Ye and Zhu [35], and Murdukhovich [36,37] have studied multiobjective optimization versions of the MPEC problem. We define the multiple objective version of the SMPEC, the SMOPEC, for $q$ objectives as

$$
\begin{aligned}
\left(\mathrm{SMOPEC}_{\Omega}\right) \min _{(x, y(\cdot))} & \left(\mathrm{E}_{\omega}\left[f_{1}(x, y(\omega), \omega)\right], \ldots, \mathrm{E}_{\omega}\left[f_{q}(x, y(\omega), \omega)\right]\right), \\
\text { s.t. } \quad x \in X, & \\
& -F(x, y, \omega) \in N_{C}(y), \quad \text { P-a.s. }
\end{aligned}
$$

We analyze the stability of weakly Pareto optimal solutions (Theorem 6.1) and of weakly Pareto stationary solutions (Theorem 6.3) below.

\subsection{Stability of Weakly Pareto-Optimal Solutions to a Convex Problem}

Let $\left\{P_{k}\right\}$ be a sequence of probability measures defined on $\mathcal{B}(\Omega)$ and consider the associated sequence of optimization problems $\left(\mathrm{SMOPEC}_{\Omega}\right)^{k}$, which are obtained from $\left(\mathrm{SMOPEC}_{\Omega}\right)$ by replacing $P$ with $P_{k}$.

Theorem 6.1 (Stability of Weakly Pareto-Optimal Solutions to $\left(\mathrm{SMOPEC}_{\Omega}\right)$ ) Let Assumption (A) hold, suppose that the mapping $F(x, \cdot, \omega)$ is strictly monotone for each $x \in X$ and $\omega \in \Omega$, that $\left(\mathrm{SMOPEC}_{\Omega}\right)$ is a convex problem, and that the sequence $\left\{P_{k}\right\}$ of probability measures weakly converges to $P$. Also, suppose that for each $k,\left(x^{k}, y_{k}(\cdot)\right)$ is a weakly Pareto-optimal solution to $\left(\mathrm{SMOPEC}_{\Omega}\right)^{k}$. Then, each limit point (there is at least one) of the sequence $\left\{\left(x^{k}, y^{k}(\cdot)\right)\right\}$ is a weakly Pareto optimal solution to $\left(\mathrm{SMOPEC}_{\Omega}\right)$.

Proof. Consider a weakly Pareto optimal solution $\left(x^{*}, y^{*}(\cdot)\right)$ to $\left(\mathrm{SMOPEC}_{\Omega}\right)$. By convexity, there exists a vector $\lambda \in \mathbb{R}_{+}^{q}$ with $\lambda_{i}>0$ for a least one $i=1, \ldots, q$, such that the solution solves the following single-objective problem [38, Prop 3.10]:

$$
\begin{aligned}
(\mathrm{S}) \min _{(x, y(\cdot))} & \sum_{i=1}^{q} \int_{\Omega} \lambda_{i} f_{i}(x, y(\omega), \omega) P(d \omega), \\
\text { s.t. } \quad & x \in X, \\
& -F(x, y, \omega) \in N_{C}(y), \quad P \text {-a.s. }
\end{aligned}
$$


Fix the vector $\lambda$ and consider a sequence of single-objective problems $(\mathrm{S})^{k}$ which are obtained from (S) by replacing $P$ with $P_{k}$.

Denote by $\left(x^{k}, y^{k}(\cdot)\right)$ an optimal solution to $(\mathrm{S})^{k}$. Since $\left(\mathrm{SMOPEC}_{\Omega}\right)$ is a convex problem, so is $\left(\mathrm{SMOPEC}_{\Omega}\right)^{k}$ for all $k$. By convexity, $\left(x^{k}, y^{k}(\cdot)\right)$ is a weakly Pareto optimal solution to $\left(\mathrm{SMOPEC}_{\Omega}\right)^{k}$ [38, Prop 3.9]. Now, apply Theorem 3.1 with the objective function $f$ replaced by

$$
f(x, y(\omega)):=\sum_{i=1}^{q} \lambda_{i} f_{i}(x, y(\omega)
$$

to get the result.

\subsection{Stability of Weakly Pareto-Stationary Solutions}

To establish stability without the assumption of convexity, we follow the development of Section 3.2 and reformulate $\left(\mathrm{SMOPEC}_{\Omega}\right)$ and $\left(\mathrm{SMOPEC}_{\Omega}\right)^{k}$ as one-level problems by treating $y$ as a function of $x$ and $\omega: y=\sigma(x, \omega)$. This is possible if, in addition to the assumptions in Theorem 6.1, Assumptions (B1) and (B2) hold. We denote the reformulations by $\left(\mathrm{SMONLP}_{\Omega}\right)$ and $\left(\mathrm{SMONLP}_{\Omega}\right)^{k}$, respectively:

$$
\begin{aligned}
&\left(\operatorname{SMONLP}_{\Omega}\right) \quad \min _{x}\left(\mathrm{E}_{\omega}\left[f_{1}(x, \sigma(x, \omega), \omega)\right], \ldots, \mathrm{E}_{\omega}\left[f_{q}(x, \sigma(x, \omega), \omega)\right]\right), \\
& \text { s.t. } \quad x \in X
\end{aligned}
$$

and

$$
\begin{aligned}
&\left(\mathrm{SMONLP}_{\Omega}\right)^{k} \quad \min _{x}\left(\mathrm{E}_{\omega}^{k}\left[f_{1}(x, \sigma(x, \omega), \omega)\right], \ldots, \mathrm{E}_{\omega}^{k}\left[f_{q}(x, \sigma(x, \omega), \omega)\right]\right), \\
& \text { s.t. } \quad x \in X .
\end{aligned}
$$

The following theorem is a KKT characterization of weak Pareto optimality for multiobjective problems due to Minami [39] and Li [40].

Theorem 6.2 Consider the problem

$$
\begin{array}{ll}
\min _{x} & \left(f_{1}(x), \ldots, f_{q}(x)\right), \\
\text { s.t. } & g_{j}(x) \leq 0, \quad j=1, \ldots, p,
\end{array}
$$

where, for each $i=1, \ldots, q, f_{i}$ is locally Lipschitz continuous, and where for each $j=$ $1, \ldots, p, g_{j} \in C^{1}$. Let the $M F C Q$ constraint qualification [Assumption (B4)] hold for all feasible solutions. Then, $x^{*}$ is a weakly Pareto optimal solution if there exist real numbers $\lambda_{i} \geq 0$ for all $i$, with $\lambda_{i}>0$ for at least one $i$, and a vector $\mu \in \mathbb{R}^{p}$ with $0^{p} \geq g\left(x^{*}\right) \perp \mu \geq 0^{p}$, such that

$$
0^{n} \in \sum_{i=1}^{q} \lambda_{i} \partial f_{i}\left(x^{*}\right)+\sum_{j=1}^{p} \mu_{j} \nabla g_{j}\left(x^{*}\right) .
$$

A solution which fulfills these conditions is called a weakly Pareto-stationary solution. Next, we establish the stability of weakly Pareto-stationary solutions. 
Theorem 6.3 (Stability of Weakly Pareto-Stationary Solutions to $\left(\mathrm{SMONLP}_{\Omega}\right)$ ) Let Assumptions (A), (B), (C2) hold, suppose that the sequence $\left\{P_{k}\right\}$ of probability measures weakly converges to $P$, and that, for each $k,\left(x^{k}, y^{k}(\cdot)\right)$ is a weakly Pareto stationary solution to $\left(\mathrm{SMONLP}_{\Omega}\right)^{k}$. Then, each limit point (there is at least one) of the sequence $\left\{\left(x^{k}, y^{k}(\cdot)\right)\right\}$ is a weakly Pareto stationary solution to $\left(\operatorname{SMONLP}_{\Omega}\right)$.

Proof. Consider a weakly Pareto-stationary solution $x^{*}$ to $\left(\operatorname{SMONLP}_{\Omega}\right)$. By Theorem 6.2 , there exist real numbers $\lambda_{i} \geq 0$ for all $i$, with $\lambda_{i}>0$ for at least one $i$, such that the conditions in Theorem 6.2 hold. Fix this value of $\lambda$, and consider a sequence $\left\{\left(x^{k}, y^{k}(\cdot)\right)\right\}$ which is Clarke stationary to $(\mathrm{S})^{k}$, that is,

$$
0^{n} \in \partial\left(\sum_{i=1}^{q} \lambda_{i} f_{i}\right)\left(x^{k}\right)+\sum_{i=1}^{p} \mu_{i} \nabla g_{i}\left(x^{k}\right) .
$$

By the properties of the generalized gradient [30, Section 2.3, Corollary 2], we have that

$$
\partial\left(\sum_{i=1}^{q} \lambda_{i} f_{i}\right)\left(x^{k}\right) \subset \sum_{i=1}^{q} \lambda_{i} \partial f_{i}\left(x^{k}\right),
$$

for any scalars $\lambda_{i}, i=1, \ldots, q$, so $\left(x^{k}, y^{k}(\cdot)\right)$ is also a weakly Pareto stationary solution to $\left(\mathrm{SMONLP}_{\Omega}\right)^{k}$. Now, apply Theorem 3.2 to the single-objective problem $(\mathrm{S})^{k}$ with the objective function $f$ replaced by

$$
f(x, y(\omega)):=\sum_{i=1}^{q} \lambda_{i} f_{i}(x, y(\omega),
$$

to get the result.

\section{Summary, Conclusions and Future Research}

Our first and main contribution in this paper is that we established that the SMPEC model is robust under the assumptions that the solution to the lower-level equilibrium problem is unique and that we have sufficient regularity conditions on the objective function and constraints. We showed that global optima as well as stationary solutions are stable with respect to changes in the probability distribution. If the SMPEC framework is used to model the problem of finding a design which should be good on average for various scenarios, then the optimal solution to SMPEC gives a design which is stable to changing conditions. The result on the robustness also gives credibility to using stochastic programming in general, since one of the criticisms on stochastic programming is that the probability distribution is often unknown or only partially known.

Our second contribution is that we have formulated, and established the robustness of solutions to, two natural extensions of the SMPEC model: first, a model where the objective is the CVaR risk measure; second, a multiobjective SMPEC model.

We also presented a discretization scheme sample average approximation (SAA), which is convergent and can be used to solve the SMPEC model. The result on the convergence 
of the SAA scheme is not new, but was included to demonstrate that the results on robustness can be combined with a method for numerically solving the SMPEC model.

The accompanying paper [6] numerically analyzes applications of the SMPEC formalism to the design of traffic networks and optimal treatment plans in intensity-modulated radiation therapy (IMRT).

Acknowledgements This work was partially financially supported by the Gothenburg Mathematical Modeling Centre (GMMC) at Mathematical Sciences (Chalmers University of Technology and the University of Gothenburg), whose main sponsor is the Swedish Foundation for Strategic Research (SSF). We thank the Associate Editor Franco Giannessi for his very encouraging and constructive remarks on a previous manuscript.

\section{References}

[1] Kall, P., Wallace, S.W.: Stochastic Programming. Wiley John \& Sons (1994)

[2] Birge, J.R., Louveaux, F.: Introduction to Stochastic Programming. Springer Series in Operations Research. Springer-Verlag, New York (1997)

[3] Ben-Tal, A., Nemirovski, A.: Robust optimization: methodology and applications. Math. Program. 92(3, Ser. B), 453-480 (2002)

[4] Zhang, Y.: General robust-optimization formulation for nonlinear programming. J. Optim. Theory Appl. 132(1), 111-124 (2007)

[5] Patriksson, M., Wynter, L.: Stochastic mathematical programs with equilibrium constraints. Oper. Res. Lett. 25(4), 159-167 (1999)

[6] Cromvik, C., Patriksson, M.: On the robustness of global optima and stationary solutions to stochastic mathematical programs with equilibrium constraints, part II: Applications. J. Optim. Theory Appl. (2009, to appear)

[7] Römisch, W.: Stability of stochastic programming problems. In: Stochastic programming, Handbooks Oper. Res. Management Sci., vol. 10, pp. 483-554. Elsevier, Amsterdam (2003)

[8] Römisch, W., Wets, R.J.B.: Stability of $\epsilon$-approximate solutions to convex stochastic programs. SIAM J. Optim. 18(3), 961-979 (2007)

[9] Heitsch, H., Römisch, W., Strugarek, C.: Stability of multistage stochastic programs. SIAM J. Optim. 17(2), 511-525 (2006)

[10] Birbil, Ş.İ., Gürkan, G., Listeş, O.: Solving stochastic mathematical programs with complementarity constraints using simulation. Math. Oper. Res. 31(4), 739-760 (2006)

[11] Shapiro, A.: Stochastic programming with equilibrium constraints. J. Optim. Theory Appl. 128(1), 223-243 (2006) 
[12] Lin, G.H., Xu, H., Fukushima, M.: Monte Carlo and quasi-Monte Carlo sampling methods for a class of stochastic mathematical programs with equilibrium constraints. Math. Methods Oper. Res. 67(3), 423-441 (2008)

[13] Evgrafov, A., Patriksson, M., Petersson, J.: Stochastic structural topology optimization: existence of solutions and sensitivity analyses. ZAMM Z. Angew. Math. Mech. 83(7), 479-492 (2003)

[14] Evgrafov, A., Patriksson, M.: Stochastic structural topology optimization: Discretization and penalty function approach. Struct. and Multidisc. Optim. 25(3), $17-188$ (2003)

[15] Evgrafov, A., Patriksson, M.: Stable relaxations of stochastic stress-constrained weight minimization problems. Struct. Multidiscip. Optim. 25(3), 189-198 (2003)

[16] Scheel, H., Scholtes, S.: Mathematical programs with complementarity constraints: stationarity, optimality, and sensitivity. Math. Oper. Res. 25(1), 1-22 (2000)

[17] Fletcher, R., Leyffer, S., Ralph, D., Scholtes, S.: Local convergence of SQP methods for mathematical programs with equilibrium constraints. SIAM J. Optim. 17(1), 259-286 (2006)

[18] Leyffer, S., López-Calva, G., Nocedal, J.: Interior methods for mathematical programs with complementarity constraints. SIAM J. Optim. 17(1), 52-77 (2006)

[19] Bard, J.F.: Practical Bilevel Optimization, Nonconvex Optimization and its Applications, vol. 30. Kluwer Academic Publishers, Dordrecht (1998)

[20] Luo, Z., Pang, J., Ralph, D.: Mathematical Programs with Equilibrium Constraints. Cambridge University Press, Cambridge (1996)

[21] Outrata, J., Kočvara, M., Zowe, J.: Nonsmooth approach to optimization problems with equilibrium constraints, Nonconvex Optimization and its Applications, vol. 28. Kluwer Academic Publishers, Dordrecht (1998)

[22] Bendsøe, M.P., Sigmund, O.: Topology Optimization. Springer-Verlag, Berlin (2003)

[23] Stackelberg, H.V.: The Theory of Market Economy. Oxford University Press, Oxford (1952)

[24] Nash, J.F.: Non-cooperative games. Ann. of Math. 54(2), 286-295 (1951)

[25] Evgrafov, A., Patriksson, M.: On the existence of solutions to stochastic mathematical programs with equilibrium constraints. J. Optim. Theory Appl. 121(1), 65-76 (2004)

[26] Lin, G.H., Chen, X., Fukushima, M.: Solving stochastic mathematical programs with equilibrium constraints via approximation and smoothing implicit programming with penalization. Math. Program. 116(1-2, Ser. B), 343-368 (2009) 
[27] Patriksson, M.: On the applicability and solution of bilevel optimization models in transportation science: A study on the existence, stability and computation of optimal solutions to stochastic mathematical programs with equilibrium constraints. Transp. Res. 42B(10), 843-860 (2008)

[28] Patriksson, M.: Robust bi-level optimization models in transportation science. Philos. Trans. R. Soc. Lond. Ser. A Math. Phys. Eng. Sci. 366(1872), 1989-2004 (2008)

[29] Robinson, S.M.: Strongly regular generalized equations. Math. Oper. Res. 5(1), 43-62 (1980)

[30] Clarke, F.H.: Optimization and Nonsmooth Analysis. Canadian Mathematical Society Series of Monographs and Advanced Texts. John Wiley \& Sons Inc., New York (1983)

[31] Borwein, J.M., Lewis, A.S.: Convex Analysis and Nonlinear Optimization. CMS Books in Mathematics/Ouvrages de Mathématiques de la SMC, 3. Springer-Verlag, New York (2000)

[32] Rockafellar, R., Uryasev, S.: Optimization of conditional value-at-risk. J. Risk 2(3), 21-41 (2000)

[33] Shapiro, A.: Monte Carlo sampling methods. In: Stochastic Programming, Handbooks Oper. Res. Management Sci., vol. 10, pp. 353-425. Elsevier, Amsterdam (2003)

[34] Shapiro, A., Xu, H.: Uniform laws of large numbers for set-valued mappings and subdifferentials of random functions. J. Math. Anal. Appl. 325(2), 1390-1399 (2007)

[35] Ye, J.J., Zhu, Q.J.: Multiobjective optimization problem with variational inequality constraints. Math. Program. 96(1, Ser. A), 139-160 (2003)

[36] Mordukhovich, B.S.: Variational Analysis and Generalized Differentiation. II, Grundlehren der Mathematischen Wissenschaften [Fundamental Principles of Mathematical Sciences], vol. 331. Springer-Verlag, Berlin (2006)

[37] Mordukhovich, B.S.: Multiobjective optimization problems with equilibrium constraints. Math. Program. 117(1-2, Ser. B), 331-354 (2009)

[38] Ehrgott, M.: Multicriteria Optimization, second edn. Springer-Verlag, Berlin (2005)

[39] Minami, M.: Weak Pareto-optimal necessary conditions in a nondifferentiable multiobjective program on a Banach space. J. Optim. Theory Appl. 41(3), 451-461 (1983)

[40] Li, X.F.: Constraint qualifications in nonsmooth multiobjective optimization. J. Optim. Theory Appl. 106(2), 373-398 (2000) 